\title{
Estado da arte da Inteligência Artifical (IA) na radiologia odontológica: revisão sistemática
}

The state of art of Artifical Intelligence (AI) in dental radiology: a systematic review El estado del arte de la Inteligencia Artifical (IA) en radiología dental: una revisión sistemática

Thaísa Pinheiro SILVA

Mirelle Nunes de CARVALHO

Wilton Mitsunari TAKESHITA

Departamento de Odontologia, Faculdade de Odontologia, Universidade Federal de Sergipe - UFS, 49060-108 Aracaju-SE, Brasil

\section{Resumo}

Introdução: Inteligência artificial (IA) é a capacidade de imitar a função do cérebro. É uma tecnologia que utiliza o aprendizado de máquina, rede neurais artificiais e a aprendizagem profunda. Ademais, utilizam de algoritmos aprimorados para "conhecer" recursos de um grande volume de dados da saúde para contribuir na atividade clínica, proporcionando um resultado mais rápido, preciso, reduzindo assim os erros de diagnóstico. Objetivo: O objetivo desta revisão sistemática é discorrer sobre o estado da arte na inteligência artificial na Radiologia Odontológica. Material e método: Na busca de evidências foram consultadas as bases de dados MEDLINE, PubMed, BBO, LILACS, BIREME, Google Acadêmico, e COCHRANE, por meio da estratégia PICOS. Todo o processo de avaliação e seleção foi executado por dois examinadores independentes. Resultados: Foram encontrados 878 artigos, seguindo os critérios de elegibilidade, os títulos e resumos foram analisados e 778 resumos excluídos do estudo, 10 textos completos, e finalmente 10 estudos foram incluídos no trabalho. Conclusão: Concluiu-se que os resultados obtidos ratificam que tanto o aprendizado profundo quanto o aprendizado de máquina e rede neurais artificiais são um campo precursor que mostram resultados animadores, principalmente pelo relevante auxilio prestado ao profissional inexperiente e por proporcionar um diagnóstico mais preciso e rápido. A inteligência artificial associada a radiologia odontológica evidencia a otimização do tempo, precisão diagnóstica, elaboração de tratamentos personalizados e previsão da eficácia no tratamento, características estas que contribuem para melhor qualidade no atendimento e, portanto, mais uma ferramenta de auxílio para os profissionais da radiologia odontológica.

Descritores: Inteligência Artificial; Aprendizagem; Aprendizado de Máquina; Radiologia.

\section{Abstract}

Introduction: Artificial intelligence $(\mathrm{Al})$ is the ability to imitate brain function. It is a technology that uses machine learning, artificial neural networks and deep learning. In addition, they use improved algorithms to "know" resources from a large volume of health data to contribute to clinical activity, providing a faster and more accurate result, thus reducing diagnostic errors. Aim: The aim of this systematic review is to discuss the state of the art in artificial intelligence in Dental Radiology. Material and method: In the search for evidence, the MEDLINE, PubMed, BBO, LILACS, BIREME, Google Scholar, and COCHRANE databases were consulted, using the PICOS strategy. The entire evaluation and selection process was carried out by two independent examiners. Results: 878 articles were found, following the eligibility criteria, the titles and abstracts were analyzed and 778 abstracts were excluded from the study, 10 full texts, and finally 10 studies were included in the work.Conclusion: It was concluded that the results obtained confirm that both deep learning and machine and artificial neural network learning are a precursor field that show encouraging results, mainly for the relevant assistance provided to inexperienced professionals and for providing a more accurate and quick diagnosis. The artificial intelligence associated with dental radiology shows the optimization of time, precision diagnostic, elaboration of personalized treatments and prediction of treatment effectiveness, characteristics that contribute to better quality of care and, therefore, another aid tool for radiology professionals.

Descriptors: Artificial intelligence; Learning; Machine Learning; Radiology.

\section{Resumen}

Introducción: La inteligencia artificial (IA) es la capacidad de imitar la función cerebral. Es una tecnología que utiliza aprendizaje automático, redes neuronales artificiales y aprendizaje profundo. Además, utilizan algoritmos mejorados para "conocer" los recursos de un gran volumen de datos de salud para contribuir a la actividad clínica, proporcionando un resultado más rápido y preciso, reduciendo así los errores de diagnóstico. Objetivo: El objetivo de esta revisión sistemática es discutir el estado del arte en inteligencia artificial en radiología dental. Material y método: En la búsqueda de evidencia, se consultaron las bases de datos MEDLINE, PubMed, BBO, LILACS, BIREME, Google Scholar y COCHRANE, utilizando la estrategia PICOS. Todo el proceso de evaluación y selección fue realizado por dos examinadores independientes. Resultados: Se encontraron 878 artículos, siguiendo los criterios de elegibilidad, se analizaron los títulos y resúmenes y se excluyeron 778 resúmenes del estudio, 10 textos completos y finalmente se incluyeron 10 estudios en el trabajo. Conclusión: Se llegó a la conclusión de que los resultados obtenidos confirman que tanto el aprendizaje profundo como el aprendizaje de máquinas y redes neuronales artificiales son un campo precursor que muestra resultados alentadores, principalmente por la asistencia relevante brindada a profesionales sin experiencia y por proporcionar un diagnóstico más preciso y rápido. La inteligencia artificial asociada con la radiología dental muestra la optimización del tiempo, la precisión diagnóstica, la elaboración de tratamientos personalizados y la predicción de la efectividad del tratamiento, características que contribuyen a una mejor calidad de la atención y, por lo tanto, otra herramienta de ayuda para los profesionales de la radiología odontológica.

Descriptores: Inteligencia Artificial; Aprendizaje; Aprendizaje Automático; Radiología.

INTRODUÇÃO

IA (Inteligência artificial) é um ramo da ciência da computação dedicado ao desenvolvimento de algoritmos de computador para realizar tarefas tradicionalmente associadas com a inteligência humana, como a capacidade de aprender e resolver problemas ${ }^{1}$.

O uso clínico da tecnologia da informação na profissão odontológica aumentou substancialmente nos últimos anos. Empregar tecnologia, especialmente a tecnologia da inteligência artificial, em áreas médicas e odontológicas pode reduzir custos, tempo, intervenção humana e erros subjetivos, além, ainda, de caracterizar a era em que se vive atualmente: Quarta Revolução Industrial ${ }^{2}$.

Por abarcar um conjunto de fenômenos como a nanotecnologia, biotecnologia, robótica, impressões 3D que somam nesta corrida 
tecnológica é que se faz necessária a busca constante por esse conhecimento para sua aplicação no dia a dia. Toda essa evolução está associada a dois grandes fatores: Capacidade de armazenamento e velocidade de processamento de informação e dados. Estes dois fatores não conseguem ser tratados eficientemente pelo intelecto humano e devido a isso a inteligência artificial é a maior das revoluções ${ }^{3}$.

A IA envolve várias etapas ou competências como reconhecer padrões e imagens, entender linguagem aberta escrita e falada, perceber relações e nexos, seguir algoritmos de decisão propostos por especialistas, ser capaz de entender conceitos, adquirir a capacidade de raciocinar pela integração de novas experiências se auto aperfeiçoando ("self learning"), resolvendo problemas ou realizando tarefas".

A combinação da IA e os Radiologistas, como uma forma de inteligência híbrida, promete alcançar níveis ainda mais altos de precisão no diagnóstico ${ }^{5}$. 0 impacto da inteligência artificial (IA) na rotina do radiologista deve ocorrer de maneira gradativa. Softwares fornecerão dados que não conseguimos extrair das imagens, e priorizarão exames de acordo com a gravidade ${ }^{6}$.

Em determinadas áreas da Radiologia, a IA já está se mostrando capaz de gerar laudos radiológicos com descrição preliminar dos achados de imagem e mensuração de algumas lesões. A IA pode detectar pequenas alterações nas imagens economizando tempo dos observadores e também pode ajudar recuperando dados prévios do paciente ou encontrar achados semelhantes em outras imagens, fornecendo uma lista de possibilidades ${ }^{1}$.

A Radiologia e o diagnóstico por imagem evoluíram substancialmente nos últimos anos e cada vez mais os Radiologistas devem se aprimorar e buscar a excelência destas áreas. É fato que a avaliação de exames como a tomografia computadorizada e a ressonância magnética apresentam desafios mesmo para especialistas experientes ${ }^{7,8}$, e, portanto, o uso das ferramentas computacionais como a inteligência artificial se torna um caminho para a expansão da Radiologia e do diagnóstico por meio de uma abordagem multidisciplinar e de alta precisão dos exames, aumentando a consistência na interpretação das imagens, auxiliando na avaliação prognóstica e apoiando o processo terapêutico de tomada de decisão ${ }^{9,10}$. Em vista disso, este trabalho tem por objetivo realizar uma revisão sistemática, e discorrer sobre o estado da arte na inteligência artificial na Radiologia Odontológica.

MATERIAL E MÉTODO

Com a proposta de responder a questão: "Qual o estado da arte da Inteligência Artificial na Radiologia Odontológica?", realizou-se uma pesquisa sistemática da literatura por meio de três etapas: Definição do objetivo; Delineamento do método de busca; Identificação na literatura, seleção dos artigos e extração dos dados. Para a identificação dos estudos incluídos na presente revisão utilizou-se a estratégia de busca "PICOS", onde $\mathrm{P}$ é a população estudada, I, a intervenção realizada, C, o tipo de teste realizado, $\mathrm{O}$, o sitio estudado e $\mathrm{S}$, o tipo de estudo para realização da pesquisa. Para a identificação dos estudos incluídos na presente revisão, uma estratégia de busca foi elaborada, a qual consiste numa combinação de palavras e termos relacionados ao assunto abordado: " $A I$ or deep learning or machine learning and Radiology and Oral or Dental or Dentistry".

Os títulos de todos os estudos encontrados como resultados das combinações dos descritores citados foram avaliados. Os estudos persistentes à exclusão pelo título tiveram os seus resumos lidos por dois examinadores independentes. Nova exclusão foi realizada e os artigos selecionados pelo resumo foram analisados por completo pelos mesmos pesquisadores. Destes, foram selecionados apenas os estudos que satisfazem claramente os critérios de inclusão. Todo o processo de seleção foi executado por dois avaliadores no ano de 2019.

Em busca da evidência, as bases de dados consultadas foram: BBO, PubMed, BIREME, Google acadêmico, e COCHRANE usando as seguintes palavras chaves: "Al", "Deep learning", "machine learning", "Radiology", "Oral","Dental", "Dentistry".

Foi necessário selecionar e revisar os estudos. Em virtude disso, foram utilizados critérios de inclusão e exclusão, para que todos os artigos, pesquisas científicas e estudos estivessem presentes na revisão sistemática, contribuindo para o conhecimento do presente tema, com as comprovações e informações recentes, que foram determinadas estudando os trabalhos a respeito do assunto.

RESULTADOS

Por meio da utilização da estratégia de busca "PICOS", e após análise de títulos e resumos, 38 artigos foram selecionados para compor a presente revisão sistemática, como mostrado na Figura 1. As principais conclusões 
dos artigos selecionados estão apresentadas na Tabela 1.

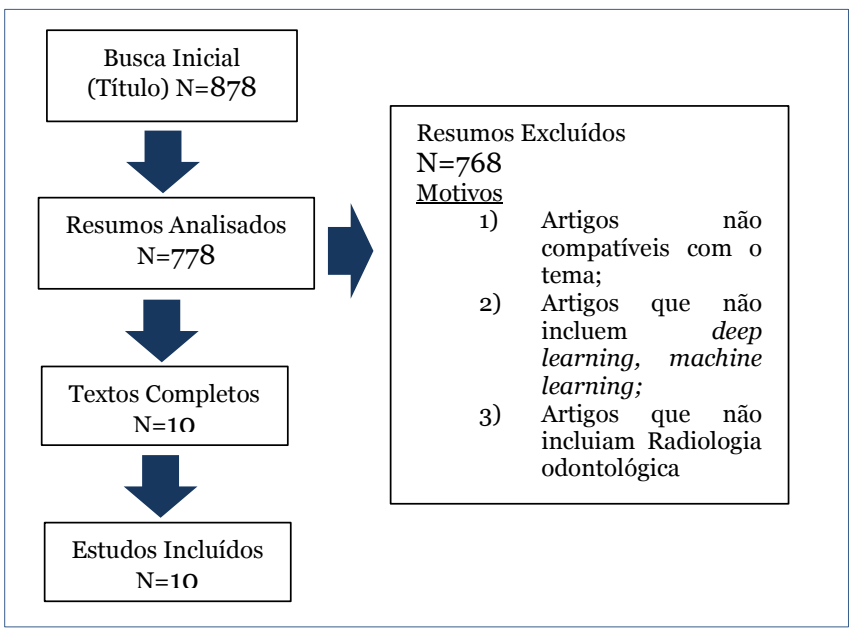

Figura 1: Fluxograma da estratégia de busca.

Tabela 1. Principais conclusões dos artigos selecionados

\begin{tabular}{|c|c|c|}
\hline Autores & Título & Conclusões \\
\hline $\begin{array}{l}\text { Liew } 5 \\
2018\end{array}$ & $\begin{array}{l}\text { The future of radiology } \\
\text { augmented with Artificial } \\
\text { Intelligence: A strategy } \\
\text { for success. }\end{array}$ & $\begin{array}{l}\text { Increased radiology increases the } \\
\text { value of radiologists economically as } \\
\text { well as socially: for our patients and } \\
\text { for the multidisciplinar, healthcare } \\
\text { team. }\end{array}$ \\
\hline $\begin{array}{l}\text { Kalappanavar et } \\
\text { al. }{ }^{11} \\
2018\end{array}$ & $\begin{array}{l}\text { Artificial intelligence: A } \\
\text { dentist's perspective }\end{array}$ & $\begin{array}{l}\text { Hence, understanding the various } \\
\text { concepts and the techniques involved } \\
\text { will have a clear advantage in the } \\
\text { future when it is time to adapt to the } \\
\text { change with redefined roles for a } \\
\text { rewarding practice. }\end{array}$ \\
\hline $\begin{array}{l}\text { Kise et al. }{ }^{12} \\
2019\end{array}$ & $\begin{array}{l}\text { A deep learning system } \\
\text { for detection of } \\
\text { Sjögren's syndrome } \\
\text { (SjS) }\end{array}$ & $\begin{array}{l}\text { The deep learning system showed a } \\
\text { high diagnostic performance for SjS, } \\
\text { suggesting that it could possibly be } \\
\text { used for diagnostic support when } \\
\text { interpreting CT images. }\end{array}$ \\
\hline $\begin{array}{l}\text { Murata et al. }{ }^{13} \\
2019\end{array}$ & $\begin{array}{l}\text { Deep-learning } \\
\text { classification using } \\
\text { convolutional neural } \\
\text { network for evaluation } \\
\text { of maxillary sinusitis on } \\
\text { panoramic radiography }\end{array}$ & $\begin{array}{l}\text { The diagnostic performance of the } \\
\text { deep-learning system for maxillary } \\
\text { sinusitis on panoramic radiographs } \\
\text { was sufficiently high. Results from } \\
\text { the deep-learning system are } \\
\text { expected to provide diagnostic } \\
\text { support for inexperienced dentists. }\end{array}$ \\
\hline $\begin{array}{l}\text { Hiraiwa et al. }{ }^{14} \\
2018\end{array}$ & $\begin{array}{l}\text { A deep-learning artificial } \\
\text { intelligence system for } \\
\text { assessment of root } \\
\text { morphology. }\end{array}$ & $\begin{array}{l}\text { The deep learning system showed high } \\
\text { accuracy in the differential diagnosis } \\
\text { of a single or extra root in the distal } \\
\text { roots of mandibular first molars. }\end{array}$ \\
\hline $\begin{array}{l}\text { Lee et al. } 15 \\
2018\end{array}$ & $\begin{array}{l}\text { Detection and diagnosis } \\
\text { of dental caries. }\end{array}$ & $\begin{array}{l}\text { A deep CNN algorithm provided } \\
\text { considerably good performance in } \\
\text { detecting dental caries in periapical } \\
\text { radiographs. }\end{array}$ \\
\hline $\begin{array}{l}\text { Kim et al. }{ }^{16} \\
2019\end{array}$ & $\begin{array}{l}\text { Deep learning-based } \\
\text { survival prediction of oral } \\
\text { cancer patients. }\end{array}$ & $\begin{array}{l}\text { A survival plan based on deep } \\
\text { apprenticeship can lead to the need } \\
\text { for guidance and guidance to doctors } \\
\text { in the selection of treatment options } \\
\text { for a better survival and prevention of } \\
\text { unnecessary treatments. }\end{array}$ \\
\hline $\begin{array}{l}\text { Shankarapillai et } \\
\text { al. }{ }^{17} \\
2010\end{array}$ & $\begin{array}{l}\text { Periodontitis Risk } \\
\text { Assessment using } \\
\text { artificial Neural Networks- } \\
\text { A Pilot Study. }\end{array}$ & $\begin{array}{l}\text { A neatly well-designed neural network } \\
\text { with the reverse propagation } \\
\text { algorithm of Levenberg Marquardt } \\
\text { can effectively be used for prediction } \\
\text { of Periodontitis Risk }\end{array}$ \\
\hline $\begin{array}{l}\text { De Tobel et al }{ }^{18} \text {. } \\
2017\end{array}$ & $\begin{array}{l}\text { An automated technique } \\
\text { to stage lower third molar } \\
\text { development on } \\
\text { panoramic radiographs. }\end{array}$ & $\begin{array}{l}\text { The overall performance of the } \\
\text { presented automated pilot technique } \\
\text { to stage lower third molar } \\
\text { development on panoramic } \\
\text { radiographs was similar to staging by } \\
\text { human observers. }\end{array}$ \\
\hline Jung et al. ${ }^{19} 2016$ & $\begin{array}{l}\text { New approach for the } \\
\text { diagnosis of extractions } \\
\text { with neural network } \\
\text { machine learning. }\end{array}$ & $\begin{array}{l}\text { Suggests that artificial intelligence } \\
\text { expert systems with neural network } \\
\text { machine learning could be useful in } \\
\text { orthodontics. }\end{array}$ \\
\hline
\end{tabular}

\section{DISCUSSÃO}

A Inteligência artificial (IA) é um avanço no campo da tecnologia que está cativando a mente dos pesquisadores em todo o mundo. Embora, de forma alguma, a IA possa substituir
- papel de um profissional, é de suma importância estar ciente das possibilidades de integrar essa tecnologia no futuro a uma prática gratificante e bem sucedida. ${ }^{11 .}$ A IA não veio para substituir o profissional, é mais uma ferramenta para aprimorar o diagnóstico.

Contrapondo-se aos achados expressivos de Kalappanavar et al. ${ }^{11}$ especialistas em Inteligência Artificial (IA) alertaram que os radiologistas podem em breve estar desempregados, um deles sendo ninguém menos que o grande mestre da aprendizagem profunda, Geo-ffrey Hinton ${ }^{5}$.

Os estudos de Kise et al. ${ }^{12}$ mostraram que o sistema de aprendizagem profunda apresentou um alto desempenho diagnóstico para a síndrome Sjögren (SjS), ao interpretar imagens de tomografia computadorizada helicodal. A precisão, sensibilidade e especificidade da aprendizagem profunda do sistema foram respectivamente $96,0 \%, 100 \%$ e $92,0 \%$. Quanto aos Radiologistas experientes, os valores correspondentes foram $98,3 \%$, $99,3 \%$ e $97,3 \%$, enquanto aqueles de Radiologistas inexperientes foram 83,5\%, 77,9\% e $89,2 \%$. Logo, o presente estudo ressaltou a importância do sistema de aprendizagem profunda como suporte diagnóstico para radiologistas inexperientes na prática clínica, visto que para o Radiologista experiente, não houve mudança significativa ao interpretar imagens TC, comparados com o sistema de aprendizagem profunda.

Murata et. al $^{13}$ também utilizaram um sistema de aprendizagem profunda em seu estudo, mas para avaliar a sinusite maxilar em radiografias panorâmicas, o qual apresentou alto desempenho, com acurácia de $87,5 \%$, sensibilidade de $86,7 \%$, especificidade de $88,3 \%$ e AUC de 0,875 . Esses valores não apresentaram diferenças significativas em comparação aos dos Radiologistas, e foram superiores aos dos residentes em odontologia. Observaram que, com o apoio do sistema DAC (detecção assistida por computador), o desempenho do diagnóstico de observadores inexperientes usando imagens panorâmicas foi melhorado à semelhança dos experientes.

Uma outra aplicação da IA está relacionada a anatomia radiográfica e suas alterações. Um fator importante que exige prudência e conhecimento profissional é que a raiz distal do primeiro molar inferior ocasionalmente pode ter uma raiz supranumerária, o que pode afetar diretamente o resultado da terapia endodôntica. Em vista disso, foi realizado um estudo de imagens de tomografia computadorizada de feixe cônico 
(TCFC) e radiografias panorâmicas, no qual 400 pacientes de 760 primeiros molares inferiores que foram analisados. Raízes distais foram examinadas em imagens de TCFC, para determinar a presença de uma raiz única ou extra. Fragmentos de imagem das raízes foram segmentados a partir de radiografias panorâmicas e aplicados a um sistema de aprendizagem profunda, e seu desempenho de diagnóstico na classificação da morfologia da raiz foi examinado. Nesse estudo, o sistema de aprendizagem profunda teve acurácia de diagnóstico de 86,9\% para determinar se as raízes distais eram únicas ou tinham raízes supranumerárias, mostrando-se altamente eficiente no diagnóstico diferencial de raiz única ou extra nas raízes distais dos primeiros molares inferiores ${ }^{14}$.

Lee et. $a 1^{15}$ reportaram um caso de algoritmos Rede neural convolucional profundos para detecção e diagnóstico de cárie dentária em radiografias periapicais. Um total de 3000 imagens radiográficas periapicais foram divididas em um conjunto de dados de treinamento e validação ( $n=2400$ [80\%]) e um conjunto de dados de teste $(n=600[20 \%])$. As precisões diagnósticas dos modelos de prémolar, molar e ambos os pré-molares e molares foram $89,0 \%(80,4-93,3), 88,0 \%(79,2-93,1)$ e $82,0 \% \quad(75,5-87,1), \quad$ respectivamente. O algoritmo de Rede neural convolucional profundo obteve uma acurácia de 0,917 (IC 95\% 0,860-0,975) no pré-molar, uma acurácia de 0,890 (IC 95\% 0,819-0,961) no molar e uma acurácia de 0,845 (IC 95\% 0,790-0,901) em ambos modelos pré-molares e molares. Dessa maneira, espera-se que os algoritmos de Rede neural convolucional estejam entre os métodos mais eficazes e eficientes para diagnosticar a cárie dentária.

No que diz respeito às redes neurais artificiais, se adequadamente treinadas podem ser um benefício para os diagnosticadores, especialmente em condições com etiologia multifatorial como a ulceração aftosa. Em estudo com 86 participantes foram usados para construir e treinar uma rede neural para prever os fatores que parecem estar relacionados à ocorrência de úlceras aftosas recorrentes. Quando isso foi testado ainda com dados não treinados de 10 participantes, os resultados revelaram previsões mais precisas tais como: sexo, hemoglobina, vitamina $B 12$ sérica, ferritina sérica, folato de glóbulos vermelhos, contagem de colônias candidíase salivar, frequência de escovação dentária e número de frutos ou os vegetais consumidos, relacionados à ulceração aftosa recorrente e apropriados para uso como dados de entrada para a construção de RNAs ${ }^{11}$. Kim et. a $1^{16}$ compararam um método de previsão de sobrevivência, baseado em aprendizagem profunda, em pacientes com carcinoma de células escamosas (CCS), e validaram seu desempenho. A predição de sobrevivência usando DeepSury, um algoritmo de predição de sobrevivência, baseado em aprendizagem profunda, foi comparado com floresta de sobrevivência aleatória (RSF) e o modelo de risco proporcional de Cox (CPH). O DeepSurv apresentou o melhor desempenho entre os três modelos. Assim, a previsão de sobrevivência baseada em aprendizagem profunda pode melhorar a precisão da previsão e orientar os médicos na escolha de opções de tratamento para uma melhor sobrevida e na prevenção de tratamentos desnecessários. Dessa forma, os autores concluíram que os pacientes se beneficiarão dessas novas técnicas, embora apenas se os clínicos as aprenderem e aplicarem.

Em relação ao aprendizado de máquina de redes neurais mostrou alto desempenho no diagnóstico de extrações, qual pode ser útil na Ortodontia para planejamento do tratamento no momento de decisão sobre as extrações e os dentes a serem removidos. Porém, a limitação deste estudo foi a ambiguidade do índice de protrusão, não pôde incluir casos com dentes perdidos, padrões de extração incomuns ${ }^{17}$.

Para Shankarapillai et. al $^{17}$ a Levenberg Marquardt, além de ser consideravelmente melhor do que o algoritmo gradiente conjugado escalado, pode ser usada como uma alternativa viável para predizer risco de futura destruição periodontal em ambientes clínicos rotineiros onde a opinião clínica especializada pode não estar prontamente disponível. Logo, uma rede neural adequadamente treinada com o algoritmo de propagação reversa de Levenberg Marquardt pode ser usada para predição do risco de periodontite, tendo em vista a capacidade comprovada de precisão, reprodutibilidade e padronização global dosistemas de aprendizado de máquina.

Com relação aos estudos de Kise et. $\mathrm{al}^{12}$, Murata et. $\mathrm{al}^{13}$, Hiraiwa et. $\mathrm{al}^{14}$ e Kim et. $\mathrm{al}^{16}$, a aprendizagem profunda se mostrou bastante positiva e de um ganho muito grande na prática clínica odontológica seja para avaliar a sinusite maxilar em radiografias panorâmicas, como na terapia endodôntica e síndrome Sjogren, bem como em pacientes com carcinoma de células escamosas (CCS). Em vista desses resultados, os profissionais que a utilizam mostram-se esperançosos no aprimoramento e detecção de outras patologias. 
Assim como Lee et. $\mathrm{al}^{15}$ e Shankarapillai et. $\mathrm{al}^{17}$, os quais utilizaram a inteligência artificial, por meio dos algoritmos, respectivamente, no diagnóstico de cárie dentária e para detectar o risco futura da destruição periodontal, mostrando-se altamente eficiente no tratamento, sendo certo que a referida tecnologia traz benefício ao paciente, reduz erros de diagnóstico e proporciona economia tempo.

A IA engloba 0 uso de algoritmos sofisticados para "aprender" recursos de um grande volume de dados de saúde e, em seguida, usar os insights obtidos para auxiliar na prática clínica; Sua configuração que pode ser equipa com habilidades de aprendizado e autocorreção para melhorar sua precisão com base no feedback; Pode ajudar a reduzir erros diagnósticos e terapêuticos que são inevitáveis na clínica humana; Extrai informações úteis de uma grande população de pacientes para auxiliar na realização de inferências em tempo real para o alerta de riscos à saúde e a previsão dos resultados de saúde ${ }^{20}$. O menor tempo para laudar exames o que implica num maior tempo para a atenção direta ao paciente também se encaixa como uma grande vantagem do uso da $\mathrm{IA}^{21}$.

A IA pode servir como uma modalidade útil no diagnóstico e tratamento de lesões da cavidade oral; Pode ser empregada na triagem e classificação de mucosa oral alterada suspeita submetida a alterações pré-malignas e malignas; Quando associada à endodontia, pode preparar mecanicamente os canais radiculares com precisão ${ }^{11}$.

São pontos positivos a precisão no diagnóstico; a padronização de procedimentos e a economia do tempo ${ }^{22}$.

No campo da ortodontia, o software pode realizar uma série de análises em radiografias e fotografias que auxiliam no diagnóstico e planejamento e no campo da implantologia e cirurgia, a Al ajudou a planejar cirurgias levando em conta os menores detalhes antes da cirurgia real $^{23}$.

Apesar dos efeitos positivos demonstrados, algumas desvantagens podem ser apontadas como a complexidade do mecanismo ${ }^{22}$, o custo envolvido em sua configuração ${ }^{23}$. Por se tratar de uma tecnologia relativamente nova, a Inteligência Artificial ainda traz questionamentos éticos, sociais e morais quanto ao seu uso e para operar e manter o funcionamento de algumas máquinas e sistemas são necessárias pessoas especializadas $^{22}$. Além disso, o pouco conhecimento dos profissionais a respeito do assunto inviabiliza a sua aplicabilidade, pois se trata de mais uma ferramenta auxiliar no diagnóstico.

CONCLUSÃO

A Inteligência artificial (IA) é um avanço no campo da tecnologia que está cativando a mente dos pesquisadores em todo o mundo. Embora, de forma alguma, a IA possa substituir - papel de um profissional, é de suma importância estar ciente das possibilidades de integrar essa tecnologia no futuro a uma prática gratificante e bem-sucedida. É necessário que o Cirurgião Dentista tenha um pleno conhecimento sobre a inteligência artificial para que possa atingir melhores resultados clínicos. Portanto, os resultados obtidos por meio desta revisão sistemática ratificam que tanto o aprendizado profundo quanto o aprendizado de máquina e rede neurais artificiais são um campo precursor que tem mostrado resultados animadores, principalmente pelo relevante auxílio prestado ao profissional na otimização do tempo, precisão diagnóstica, elaboração de tratamentos personalizados e previsão de eficácia no tratamento, características estas que contribuem para melhor qualidade no atendimento e, portanto, grande impacto em sua significância clínica.

REFERÊNCIAS

1. Yaji A, Prasad S, Pai A. Artificial Intelligence in Dento-Maxillofacial Radiology. Act Scient Dent Scienc. 2018;3 (1):116-18.

2. Khanna SS, Dhaimade PA. Artificial Intelligence: Transforming Dentistry Today. J Ind Basic Ap Med Research. 2017;6(3):161-64.

3. Schwab KA Quarta revolução industrial. Barcelona: Debate; 2016.

4. Lobo LC. Artificial Intelligence, the Future of Medicine and Medical Education. Rev Bras Educ Med. 2018;42(3):3-8.

5. Liew $\mathrm{C}$. The future of radiology augmented with Artificial Intelligence: A strategy for success. Eur J Radiol. 2018;102:152-56.

6. Paiva OA, Prevedello LM. O potencial impacto da inteligência artificial na radiologia. Radiol Bras. 2017; 50(5):V-VI.

7. Azevedo-Marques PM, Mencattini A, Salmeri R, et al. Medical image analysis and informatics. Boca Raton, FL: CRC Press; 2017.

8. Doi K. Computer-aided diagnosis in medical imaging: historical review, current status and future potential. Comput Med Imaging Graph. 2007;31:198-211

9. Aerts HJWL. The potential of radiomic-based phenotyping in precision medicine: a review. JAMA Oncol. 2016;2:1636-42.

10. Keek SA, Leijenaar RT, Jochems A, et. al. A review on radiomics and the future of 
theranostics for patient selection in precision medicine. Br J Radiol. 2018;91:20170926

11. Kalappanavar A, Sneha S, Annigeri RG. Artificial intelligence: A dentist's perspective - J Med RadiolPathol Surg. 2018;5:2-4.

12. Kise $Y$, Ikeda $H$, Fujii $T$, Fukuda M, Ariji $Y$, Fujita $\mathrm{H}$, Katsumata A, Ariji E. Preliminary study on the application of deep learning system to diagnosis of Sjögren's syndrome on CT images. Dentomaxillofac Radiol. 2019;48(6):20190019.

13. Murata M, Ariji Y, Ohashi Y, Kawai T, Fukuda M, Funakoshi T, Kise Y, Nozawa M, Katsumata A, Fujita H, Ariji E. Deep-learning classification using convolutional neural network for evaluation of maxillary sinusitis on panoramic radiography. Oral Radiol. 2019;35(3):301-7.

14. Hiraiwa T, Ariji Y, Fukuda M, Kise Y, Nakata K, Katsumata A, Fujita H, Ariji E. A deep-learning artificial intelligence system for assessment of root morphology of the mandibular first molar on panoramic radiography. Dentomaxillofac Radiol. 2019;48(3):20180218.

15. Lee JH, Kim DH, Jeong SN, Choi SH. Detection and diagnosis of dental caries using a deep learning-based convolutional neural network algorithm. J Dent. 2018: 10.1016/j.jdent. 2018.07.015.

16. Kim, D.W., Lee, S., Kwon, S, Nam W, Cha IH, Kim HJ. Deep learning-based survival prediction of oral cancer patients. Sci Rep. 2019;9:6994.

17. Shankarapillai R, Mathur Ik, Nair MA, Rai N, Mathur A. Periodontitis Risk Assessment using two artificial Neural Networks-A Pilot Study. Int J Dent Clin. 2010:2(4):36-40

18. De Tobel J, Radesh P, Vandermeulen D, Thevissen PW. An automated technique to stage lower third molar development on panoramic radiographs for age estimation: a pilot study. J Forensic Odontostomatol. 2017; 35(2):42-54.

19.Jung SK, Kim TW. New approach for the diagnosis of extractions with neural network machine learning. Am $\mathrm{J}$ Orthod Dentofacial Orthop. 2016;149(1):127-33.

20. Jha S, Topol EJ. Information and Artificial Intelligence. Journal of the American College of Radiology.2018;15(3):509-11.

21. Majumdar B, Sarode SC, Sarode GS, Patil S. Technology: Artificial intelligence. J Brit Dent. 2018;224(12):916.

22. Marques JR. Inteligência Artificial: vantagens e desvantagens quanto ao seu uso. Portal IBC2017. [Citado 2019 Fev 4]. Disponível em : https://www.ibccoaching.com.br/portal/artigos/in teligencia-artificial-vantagens-desvantagensquanto-seu-uso/

23. Khanna SS, Dhaimade P.A. Artificial Intelligence: Transforming Dentistry Today. J Ind Basic Ap Med Research. 2017;6(3):161-64.
CONFLITO DE INTERESSES

Os autores declaram não haver conflitos de interesse

AUTOR PARA CORRESPONDÊNCIA

\section{Thaísa Pinheiro Silva}

Rua José Deodoro dos Santos, $n^{\circ} 170$,

Luzia, Repletto Condomínio Club, Ed. Arbore, Ap. 1405 49048-390 Aracaju - SE, Brasil

E-mail: thaisapinheirosilva@hotmail.com

Submetido em 13/06/2020

Aceito em 23/10/2020 